\title{
On Lempel-Ziv Complexity of Sequences
}

\author{
Ali Doğanaksoy ${ }^{1,2,4}$ and Faruk Göloğlu $u^{2,3}$ \\ ${ }^{1}$ Department of Mathematics, Middle East Technical University \\ Ankara, Turkey \\ aldoks@metu.edu.tr \\ ${ }^{2}$ Institute of Applied Mathematics, Middle East Technical University \\ Ankara, Turkey \\ ${ }^{3}$ Dept. of Computer Technology and Information Systems \\ Bilkent University, Ankara, Turkey \\ gologlu@bilkent.edu.tr \\ 4 TUBITAK-UEKAE, Gebze, Turkey
}

\begin{abstract}
We derive recurrences for counting the number $a(n, r)$ of sequences of length $n$ with Lempel-Ziv complexity $r$, which has important applications, for instance testing randomness of binary sequences. We also give algorithms to compute these recurrences. We employed these algorithms to compute $a(n, r)$ and expected value, $E P_{n}$, of number of patterns of a sequence of length $n$, for relatively large $n$. We offer a randomness test based on the algorithms to be used for testing randomness of binary sequences. We give outputs of the algorithms for some $n$. We also provide results of the proposed test applied to the outputs of contestant stream ciphers of ECRYPT's eSTREAM.
\end{abstract}

Keywords: Lempel-Ziv complexity, randomness, $\chi^{2}$-statistics.

\section{Introduction}

There are several complexity measures to test the randomness of a sequence. Linear complexity, for example, is one of these measures. Lempel-Ziv complexity of a sequence was defined by Lempel and Ziv in 1976 [1]. This measure counts the number of different patterns in a sequence when scanned from left to right. For instance Lempel-Ziv complexity of $s=101001010010111110$ is 8, because when scanned from left to right, different patterns observed in $s$ are $1|0| 10|01| 010|0101| 11|110|$.

Lempel-Ziv complexity is the basis of LZ77 compression algorithm [2]. It is also an important measure used in cryptography. For instance, it was used to test the randomness of the output of a symmetric cipher [3]. One expects a 'random' sequence of length $n$ has a close Lempel-Ziv complexity to the expected value of Lempel-Ziv complexity of a sequence of length $n$. However, the expected value of Lempel-Ziv complexity for arbitrary $n$ is unknown. For limiting behaviour of this value, the reader is referred to Jacquet and Szpankowski [4] and Kirschenhofer et. al. [6]. Some cryptographic applications of Lempel-Ziv complexity are given in $[5]$.

G. Gong et al. (Eds.): SETA 2006, LNCS 4086, pp. 180-189, 2006.

(C) Springer-Verlag Berlin Heidelberg 2006 
Some sequences end with a pattern that was observed before (one simplest example is: $s=0 \mid 0$ ), which we call open; and remaining sequences (i.e., that end without same pattern appearing twice) are called closed.

In this paper we derive a recurrence for $a(n, r)$, the number of sequences of length $n$ with Lempel-Ziv complexity $r$; and a recurrence for $c(n, r)$, the number of closed sequences of length $n$ with Lempel-Ziv complexity $r$. By using these recurrences and with the help of a computer, we compute $a(n, r)$ for as large $n$ as possible.

A test based on Lempel-Ziv complexity was used in the NIST test suite, to test the randomness of sequences. However the test had some weaknesses. First of all, the test could only be applied to data of a specified length: $10^{6}$ bits. Moreover, the test used empirical data generated by SHA-1 (under randomness assumptions) for estimating the expected value of Lempel-Ziv complexity of sequences of length $10^{6}$ bits. Apparently, the data generated by SHA-1 led to not-so-good an estimate, hence, for instance, first $10^{6}$ bits of the binary expansion of $e$ failed the randomness test. Using asymptotic formulae for an estimate will not work either, since the sequences, as we will see in the forthcoming sections, are distributed tightly around the mean. Recently, apparently because of the spelt out reasons, Lempel-Ziv test had been excluded from the NIST test suite. Inclusion of a Lempel-Ziv complexity based randomness test in a statistical test suite is important concerning completeness. In the last section, we offer a new and stronger variant of this test, which employs the results we found and present in this paper. The data we use are neither empirical nor derived from asymptotic formulae, but are exact results; thanks to the recurrences (1),(2), hence avoid the errors present in the previous test.

\section{Preliminaries}

Lempel-Ziv complexity was first defined in [1]. We include the definitions here. For the sequel, juxtaposition denotes concatenation of strings.

Let $p=p_{1} p_{2} \cdots p_{k}$ and $s=s_{1} s_{2} \cdots s_{k} \cdots s_{n}$ be binary strings. $p$ is a prefix of $s$ if $p_{i}=s_{i}$ for $1 \leq i \leq k$. If $k<n$, then $p$ is said to be a proper prefix of $s$.

Let again $s=s_{1} s_{2} \cdots s_{n}$ be a binary string of length $n . \sigma_{1}|\cdots| \sigma_{r}$ is called the Lempel-Ziv partition of $s$, if

- for $1 \leq i<r, \sigma_{i}$ is different from $\sigma_{j}$ for $0 \leq j<i$, satisfying

$-s=\sigma_{1} \sigma_{2} \cdots \sigma_{r}$, and

- for $1 \leq i \leq r$, every proper prefix of $\sigma_{i}$ is equal to $\sigma_{j}$ for some $0 \leq j<i$.

where $\sigma_{i}$ are binary strings (patterns) and $\sigma_{0}$ is defined to be the empty string.

Lempel-Ziv complexity of $s$ is then defined to be the number of patterns, $r$, in the Lempel-Ziv partition of $s$.

Note that $\sigma_{r}$ may or may not satisfy $\sigma_{r}=\sigma_{i}$ for some $1 \leq i<r$. If $\sigma_{r}=\sigma_{i}$ for some $1 \leq i<r$, then we call $s$ an open sequence. $s$ is called closed otherwise.

Lempel-Ziv partition of:

- an open sequence $s$ is denoted by $s=\sigma_{1}|\cdots| \sigma_{r}$,

- a closed sequence $s$ is denoted by $s=\sigma_{1}|\cdots| \sigma_{r} \mid$. 
Succint background for statistical tests (especially for randomness) can be found in $[3]$.

\section{The Recurrences}

Let $A(n, r)$ denote the set of binary strings of length $n$ with Lempel-Ziv complexity $r$. For any $s=s_{1} \cdots s_{n} \in A(n, r)$ and $s_{n+1} \in\{0,1\}$, it is evident that $s s_{n+1} \in A(n+1, r) \cup A(n+1, r+1)$. In fact $s 0 \in A(n+1, r) \Longleftrightarrow s 1 \in A(n+1, r)$. We define

$$
C(n, r)=\{s \in A(n, r): s 0 \in A(n+1, r+1)\} .
$$

Note that $C(n, r)$ is the set of closed sequences. One has

$$
a(n, r)=2 c(n-1, r-1)+2[a(n-1, r)-c(n-1, r)],
$$

where $a(n, r)=|A(n, r)|$ and $c(n, r)=|C(n, r)|$.

Given $s=s_{1} \cdots s_{n} \in C(n, r)$, let $\sigma_{1}|\ldots| \sigma_{r} \mid$ be the Lempel-Ziv partition of $s$. We define the mapping $\delta_{n, r}^{0}: C(n, r) \rightarrow C(n+r+1, r+1)$ by setting $\delta_{n, r}^{0}(s)=00 \sigma_{1} 0 \sigma_{2} \cdots 0 \sigma_{r}$ for $s=\sigma_{1} \cdots \sigma_{r} \in C(n, r) . \delta_{n, r}^{1}$ is defined in a similar way. Let $C_{0}(n, r)=\operatorname{Im}\left(\delta_{n-r, r-1}^{0}\right), C_{1}(n, r)=\operatorname{Im}\left(\delta_{n-r, r-1}^{1}\right)$, and $C_{*}(n, r)=$ $C_{0}(n, r) \cup C_{1}(n, r)$. It follows that $c_{*}(n, r)=c_{0}(n, r)+c_{1}(n, r)=2 c(n-r, r-1)$, where $c_{*}(n, r)=\left|C_{*}(n, r)\right|, c_{0}(n, r)=\left|C_{0}(n, r)\right|, c_{1}(n, r)=\left|C_{1}(n, r)\right|$, and $\operatorname{Im}(f)$ denotes the image of the map $f$.

Any $s=\sigma_{1}|\cdots| \sigma_{r} \mid \in C(n, r) \backslash C_{*}(n, r)$ has a unique substring $\alpha=\alpha_{1}|\cdots| \alpha_{p} \mid$ $\in C_{0}(a, p)$, and a unique substring $\beta=\beta_{1}|\cdots| \beta_{q} \mid \in C_{1}(b, q)$ such that $a+b=n$ and $p+q=r$.

For any pair $(p, q)$ of positive integers, we consider the subset $\Xi^{p, q}$ of the symmetric group $S^{p+q}$ given by:

$$
\Xi^{p, q}=\left\{\sigma \in S^{p+q}: i<j \leq p \text { or } p+1 \leq i<j \Rightarrow \sigma(i)<\sigma(j)\right\} .
$$

For $\alpha=\alpha_{1}|\cdots| \alpha_{p}\left|\in C_{0}(a, p), \beta=\beta_{1}\right| \cdots\left|\beta_{q}\right| \in C_{1}(b, q)$ and $\pi \in \Xi^{p, q}, \pi(\alpha, \beta)$ stands for $\pi\left(\alpha_{1}, \ldots, \alpha_{p}, \beta_{1}, \ldots, \beta_{q}\right)$.

Any triple $(\pi, \alpha, \beta)$, where $\pi \in \Xi^{p, q}, \alpha \in C_{0}(a, p), \beta \in C_{1}(b, q)$, corresponds to a unique string in $C(n, r) \backslash C_{*}(n, r)$, namely to $\pi(\alpha, \beta)$. Conversely given any $\sigma \in C(n, r) \backslash C_{*}(n, r)$, there exist a unique triple $(\pi, \alpha, \beta)$, such that $\pi(\alpha, \beta)=\sigma$.

Given $a, b, p$ and $q$, the number of all possible triples $(\pi, \alpha, \beta)$ with $\pi \in$ $\Xi^{p, q}, \alpha \in C_{0}(a, p), \beta \in C_{1}(b, q)$ is

$$
\left(\begin{array}{c}
p+q \\
p
\end{array}\right) c_{0}(a, p) c_{1}(b, q) .
$$

It follows that

$$
\begin{aligned}
c(n, r)-c_{*}(n, r) & =\sum_{a+b=n} \sum_{\substack{p+q=r \\
p, q \geq 1}}\left(\begin{array}{c}
p+q \\
p
\end{array}\right) c_{0}(a, p) c_{1}(b, q) \\
& =\sum_{a+b=n} \sum_{\substack{p+q=r \\
p, q \geq 1}}\left(\begin{array}{c}
p+q \\
p
\end{array}\right) c(a-p, p-1) c(b-q, q-1)
\end{aligned}
$$




$$
\begin{aligned}
\Rightarrow c(n, r)= & 2 c(n-r, r-1)+ \\
& \sum_{\substack{a+b=\\
n}} \sum_{\substack{p+q=r \\
p, q \geq 1}}\left(\begin{array}{c}
p+q \\
p
\end{array}\right) c(a-p, p-1) c(b-q, q-1) \\
= & 2 c(n-r, r-1)+ \\
& \sum_{0 \leq a \leq n} \sum_{1 \leq p<r} \underbrace{\left(\begin{array}{l}
r \\
p
\end{array}\right) c(a-p, p-1) c(n-a-r+p, r-p-1)}_{\tau(n, r, a, p)}
\end{aligned}
$$

We can give upper and lower bounds for $r$, since not all $r$ are possible given any $n$. Indeed, observing $s=0|00| 000 \mid \cdots$ has minimum complexity, and

$$
s=0|1| 00|01| 10|11| 000|001| 010|011| \cdots
$$

has maximum complexity among all sequences of length $n$, we limit $r$ by:

$$
\left\lceil\frac{-1+\sqrt{1+8 n}}{2}\right\rceil \leq r \leq\left\lceil\frac{2^{t+2}+n-2 t-4}{t+1}\right\rceil
$$

where $t=\max \left\{i \in \mathbb{N}:(i-1) 2^{i+1}+2 \leq n\right\}$. Note here that $r$ is bounded by $r<k \frac{n}{\log n}$, for some $k \in \mathbb{N}$. Indeed, $t<\log n$ for all $n \geq 2$. Also

$$
\left\lceil\frac{2^{t+2}+n-2 t-4}{t+1}\right\rceil=\left\lceil\frac{2^{t+2}-2(t+2)}{t+1}+\frac{n}{t+1}\right\rceil
$$

increases when $t$ increases, hence

$$
r \leq\left\lceil\frac{2^{t+2}+n-2 t-4}{t+1}\right\rceil \leq\left\lceil\frac{4 \cdot 2^{\log n}+n-2 \log n-4}{\log n+1}\right\rceil<5 \frac{n}{\log n}
$$

\section{Algorithms and Their Complexities}

(1) implies computing $c(n, r)$ for all $k \leq n$, and knowing $a(1,1)=2$, is enough to compute $a(n, r)$ for any $n \geq 2$. Therefore we use (2) to compute $c(n, r)$, the result of which is used by another algorithm to compute $a(n, r)$. However, it is inefficient to compute larger values (e.g., computing $a(2000, r)$ for all $r$ takes two hours on a standard PC with our implementation). We use the recurrence (2) in the following algorithm.

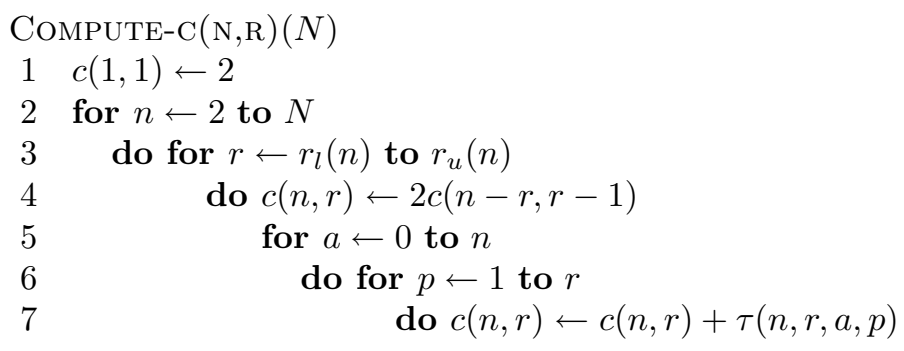


After we compute all $c(n, r)$ for $n<N$, we use the following algorithm which is based on the recurrence (1) to compute $a(n, r)$.

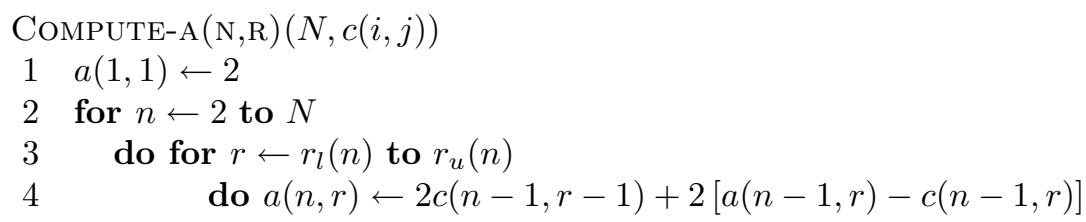

In the algorithms, $r_{l}$ and $r_{u}$ are computed by the inequalities (3).

We have the following observations for the complexity of the algorithms. For any $(n, r)$ pair, $c(n, r)<2^{n}$, hence an (at most) $n$-bit integer. Since $r \leq k$. $n / \log n$, and complexity of multiplication of two $n$ bit integers is $\mathcal{O}(n \log n)$ we have :

Proposition 1. Complexity of the algorithm:

- Compute- $\mathrm{C}(\mathrm{N}, \mathrm{R})$ is $\mathcal{O}\left(n^{5} / \log n\right)$, and

- Compute-A $(\mathrm{N}, \mathrm{R})$ is $\mathcal{O}\left(n^{2} / \log n\right)$ (after computing $c(n, r)$ ).

\section{Computing $a(n, r)$ for Large $n$}

Tables 1 and 2 in Appendix A display the results for $n=100$ and $n=250$. Note that without using the recurrences (1) and (2), time complexity to find these results is $\mathcal{O}\left(n 2^{n-1}\right)$, impractical for today's computers for $n=100$ or $n=250$.

Expected values $E P_{n}$ of number of patterns of a sequence of length $n$, for $n=100$ and $n=250$ are $E P_{100}=29.04319$ and $P_{250}=57.93485$.

Table 4 in Appendix C displays the $E P_{n}$ values for some $n \leq 1000$.

\section{An Application: A Randomness Test for Binary Sequences}

We design a randomness test for binary sequences which employs the algorithms as follows.

Given a sequence of length $n$ bits. First divide the sequence into $M=\left\lfloor\frac{n}{k}\right\rfloor$ non-overlapping blocks of length $k$ bits, omitting if necessary last few bits. Apply Lempel-Ziv partitioning procedure to each of these $M$ blocks to get the number of Lempel-Ziv partitions $\pi_{i}$ for $1 \leq i \leq M$. From now on we choose $k=1024$. Set:

$$
\begin{aligned}
& r_{1}=\left|\left\{i: \pi_{i} \leq 174,1 \leq i \leq M\right\}\right|, \\
& r_{2}=\left|\left\{i: \pi_{i}=175,1 \leq i \leq M\right\}\right|, \\
& r_{3}=\left|\left\{i: \pi_{i}=176,1 \leq i \leq M\right\}\right|, \\
& r_{4}=\left|\left\{i: \pi_{i}=177,1 \leq i \leq M\right\}\right|, \\
& r_{5}=\left|\left\{i: \pi_{i} \geq 178,1 \leq i \leq M\right\}\right| .
\end{aligned}
$$


We obviously have $\sum_{i=1}^{5} r_{i}=M$. The numbers 174 through 178 are chosen to align $E P_{1024}=176.09949$ to the center.

Define the random variable $X$ to be the number of partitions of a random sequence of fixed length $k$ bits. Employing the algorithm described in Section 4, we obtain the following probabilities for $k=1024$.

$$
\begin{aligned}
& p_{1}=\operatorname{Pr}(X \leq 174)=0.05262, \\
& p_{2}=\operatorname{Pr}(X=175)=0.19987, \\
& p_{3}=\operatorname{Pr}(X=176)=0.39720, \\
& p_{4}=\operatorname{Pr}(X=177)=0.29107, \\
& p_{5}=\operatorname{Pr}(X \geq 178)=0.05924 .
\end{aligned}
$$

Then apply the $\chi^{2}$-statistic to the observed data:

$$
X(o b s)=\sum_{i=1}^{5} \frac{\left(r_{i}-M p_{i}\right)^{2}}{M p_{i}}
$$

to get the $\chi^{2}$ random variable $X(o b s)$ with degree of freedom 4 . Then, the $P$ value of the test is:

$$
\frac{\int_{X(o b s)}^{\infty} e^{-u / 2} u d u}{\Gamma(2) 2^{2}}=\frac{1}{2}(X(o b s)+2) e^{-X(o b s) / 2} .
$$

A condition that can be safely used with $\chi^{2}$-approximation is:

$$
M \cdot \min \left\{p_{i}: 1 \leq i \leq 5\right\}=M \cdot 0.05262 \geq 5 \text {. }
$$

Hence, if $k$ is chosen to be 1024 , then $n$ should satisfy $n \geq 100000$ approximately. Note that the test can be applied for any $k$ with respective $p_{i}$ 's and 'bins' are aligned around $E P_{k}$ and of course provided that computation of $a(k, l)$ is feasible.

If the $P$-value of the observed data is less than some threshold (e.g., 0.01), one can conclude that the given sequence is not random. The test applied to the outputs of stream ciphers contesting in ECRYPT's eSTREAM can be found in Appendix B.

\section{Conclusion and Future Work}

We give two recurrences for the number of sequences of length $n$ with Lempel-Ziv complexity $r$. We also give the the algorithms and the output of the computer programs that we run to calculate $a(n, r)$ for relatively large values.

We also offer a randomness test that can be applied to the output of ciphers.

The recurrence (2) is quite hard to simplify, but can be used to improve the limiting behaviour of the expected value of $a(n, r)$. 


\section{Acknowledgments}

The authors would like to thank to anonymous refeeres for their comments, which improved the presentation of the paper. The authors also would like to thank Meltem Sönmez Turan and Çă̆daş Çalık for making the outputs of eSTREAM contestant stream ciphers available.

\section{References}

1. Lempel, A., Ziv, J.: On the complexity of finite sequences. IEEE Transactions on Information Theory IT-22 (1976) 75-81

2. Ziv, J., Lempel, A.: A universal algorithm for sequential data compression. IEEE Transactions on Information Theory IT-23 (1977) 337-343

3. Soto, J.: Statistical testing of random number generators. In: Proceedings of the 22nd National Information Systems Security Conference, Crystal City, Virginia (1999)

4. Jacquet, P., Szpankowski, W.: Asymptotic behavior of the Lempel-Ziv parsing scheme and digital search trees. Theoretical Computer Science 144 (1995)

5. Mund, S.: Ziv-Lempel complexity for periodic sequences and its cryptographic application. In: Advances in cryptology - EUROCRYPT 91 (Brighton, 1991). Volume 547 of Lecture Notes in Comput. Sci. Springer, Berlin (1991) 114-126

6. Kirschenhofer, P., Prodinger, H., and Szpankowski, W.: Digital Search Trees Again Revisited: The Internal Path Length Perspective, SIAM Journal on Computing 23 (1994) 598-616

\section{A Tables for $n=100$ and $n=250$}

Table 1. $a(100, r)$ and their probabilities

\begin{tabular}{l|l|l}
$\mathbf{r}$ & $\mathbf{a}(\mathbf{1 0 0}, \mathbf{r})$ & $\mathbf{a}(\mathbf{1 0 0}, \mathbf{r}) / \mathbf{2}^{\mathbf{1 0 0}}$ \\
\hline 14 & 122880 & 0.000000000000000 \\
15 & 96129024 & 0.000000000000000 \\
16 & 1754408140 & 0.000000000000000 \\
17 & 169010698649 & 0.000000000000000 \\
18 & 12282745099264 & 0.000000000000000 \\
19 & 726896570696704 & 0.000000000000006 \\
20 & 35864704163873996 & 0.000000000000283 \\
21 & 1555171539525474304 & 0.000000000012268 \\
22 & 629504083451115732992 & 0.000000000496591 \\
23 & 23657061862581861351424 & 0.000000018662131 \\
24 & 796717339700675605430272 & 0.000000628499162 \\
25 & 25016712354109852183691264 & 0.000019734706353 \\
26 & 701956405285233154502688768 & 0.000553745965299 \\
27 & 14929637765344244033503887360 & 0.011777407562191 \\
28 & 190072463603886098540862111744 & 0.149940735696149 \\
29 & 785071700104053917078962307072 & 0.619312372007483 \\
30 & 276807820976750678936983175168 & 0.218362868227975 \\
31 & 41183640732091617843871744 & 0.000032488164108
\end{tabular}


Table 2. $a(250, r)$

\begin{tabular}{l|l}
$\mathbf{r}$ & $\mathbf{a}(\mathbf{2 5 0}, \mathbf{r})$ \\
\hline 22 & 12582912 \\
23 & 172462440448 \\
24 & 207405092700160 \\
25 & 100022234734919680 \\
26 & 29027442465801502720 \\
27 & 5970493862438356647936 \\
28 & 947059437548499752058880 \\
29 & 124084577391675511972954112 \\
30 & 14104448150286646440414281728 \\
31 & 1429659188269782925153552039936 \\
32 & 131701470381268947695969402486784 \\
33 & 11234825836624304676748166609502208 \\
34 & 902346385748231250614173057894580224 \\
35 & 68848617082812392433571189369104498688 \\
36 & 5026197932887293151555523266808542920704 \\
37 & 353853624800555379505246051484079264628736 \\
38 & 24168383146155367527845519053853996700663808 \\
39 & 1608511050085914176405326626207802044763340800 \\
40 & 104662286330519094422345952269389211024618422272 \\
41 & 6671326955511762120610779318782504320898951020544 \\
42 & 417414764650712462333990517379047167232803455631360 \\
43 & 25695057332640828405359742259152343883668370141216768 \\
44 & 1557264023287411624909081480426191697539573325326450688 \\
45 & 92701547946190343870914352507209237010079274453119795200 \\
46 & 5404237040271065934800659259750349232918183084398749941760 \\
47 & 308137037472343306269203510492671163205021043338817023508480 \\
48 & 17174851395953502738636183446022389293687597007698530091925504 \\
49 & 931658807304593772659970661068671319161079379311405098319478784 \\
50 & 48481739469168604779398196043362721869737926469656763302282264576 \\
51 & 2362999038927091779739893669395333742467402505543139386088962916352 \\
52 & 104713381850515314827585466063284598037259318649923004999242356883456 \\
53 & 4060841943120707511367011625729606171901794317261167326529027544449024 \\
54 & 130173461076947361401256777713070992922594587328928989706197194351050752 \\
55 & 3144432233774945014197088996141663864291615518236615683726382172345466880 \\
56 & 49270065837659893327338857961415416600618856787734271093560018969747783680 \\
57 & 389584816963822529432810975258074767638122905768577642480619865183753338880 \\
58 & 990152575211374388394386838415206581167171380538108666357249216731099955200 \\
59 & 373709741973491221158081813947703844668617084164776240113666220254822400000 \\
60 & 3255419797444187375980631332217791151748569890952982076721561862144000000 \\
61 & 886666544515752607846923627683910176444275554890872258560000000000
\end{tabular}




\section{B Test Results}

Table 3. Results of LZ randomness test applied to eSTREAM contestants with parameters $M=800, k=1024$ and threshold $<0.01$

\begin{tabular}{l|l} 
eSTREAM stream cipher & P - value \\
\hline ABC-v2 & 0.215686 \\
ACHTERBAHN & 0.856026 \\
CryptMT & 0.281958 \\
DECIM & 0.435354 \\
DICING & 0.391681 \\
Dragon & 0.784314 \\
Edon80 & 0.958401 \\
F-FCSR-8 & 0.559503 \\
FUBUKI & 0.805604 \\
Frogbit & 0.247524 \\
Grain & 0.092822 \\
HC-256 & 0.189772 \\
Hermes8 & 0.548511 \\
LEX & 0.192730 \\
MAG & 0.172511 \\
MICKEY-128 & 0.951844 \\
MICKEY & 0.958706 \\
Mir-1 & 0.624140 \\
POMARANCH & 0.864929 \\
Phelix & 0.422482 \\
Polar-Bear & 0.032209 \\
ProVEST-4 & 0.902847 \\
Py & 0.518629 \\
Rabbit & 0.654306 \\
SFINKS & 0.327318 \\
Salsa20 & 0.325591 \\
TRIVIUM & 0.624686 \\
TSC-3 & 0.943600 \\
WG & 0.836510 \\
Yamb & 0.514665 \\
ZK-Crypt & 0.590525 \\
&
\end{tabular}




\section{Table of Expected Values}

Table 4. Expected values $E P_{n}$ for some $n \leq 1000$

\begin{tabular}{l|l}
$\mathbf{n}$ & $\mathbf{E P}_{\mathbf{n}}$ \\
\hline 968 & 168.285154708125871909 \\
969 & 168.425325208575350399 \\
970 & 168.565472359678417715 \\
971 & 168.705595531148748041 \\
972 & 168.845694563342602216 \\
973 & 168.985769897582357720 \\
974 & 169.125822268928967939 \\
975 & 169.265852191319223625 \\
976 & 169.405859611321451745 \\
977 & 169.545843978226506736 \\
978 & 169.685804672692994605 \\
979 & 169.825741477831057654 \\
980 & 169.965654751385158620 \\
981 & 170.105545179628954902 \\
982 & 170.245413297909854937 \\
983 & 170.385259128160740312 \\
984 & 170.525082193178254703 \\
985 & 170.664881890971218212 \\
986 & 170.804657952890038172 \\
987 & 170.944410653561969008 \\
988 & 171.084140625407537432 \\
989 & 171.223848418188401220 \\
990 & 171.363534125420147438 \\
991 & 171.503197345751651805 \\
992 & 171.642837501466368323 \\
993 & 171.782454278665110691 \\
994 & 171.922047870128312202 \\
995 & 172.061618849379214303 \\
996 & 172.201167772807546273 \\
997 & 172.340694800677496380 \\
998 & 172.480199609262570074 \\
999 & 172.619681652361296729 \\
1000 & 172.759140578329111086
\end{tabular}

